\title{
Coronagraphic search for exo-planets with a hypertelescope
}

\section{In the thermal IR ${ }^{\star}$}

\author{
P. Riaud ${ }^{1,2}$, A. Boccaletti ${ }^{1}$, S. Gillet ${ }^{2,5}$, J. Schneider ${ }^{3}$, A. Labeyrie ${ }^{2,5}$, L. Arnold ${ }^{4}$, J. Baudrand ${ }^{1}$, O. Lardière ${ }^{2,5}$, \\ J. Dejonghe ${ }^{2,5}$, and V. Borkowski ${ }^{2,5}$ \\ 1 LESIA, Observatoire de Paris-Meudon, 5 place J. Janssen, 92195 Meudon, France \\ 2 LISE-Observatoire de Haute-Provence, 04870 St Michel l'Observatoire, France \\ e-mail: sgohp@obs-hp.fr; lardiere@obs-hp.fr; dejonghe@obs-hp.fr \\ 3 LUTH, Observatoire de Paris-Meudon, 5 place J. Janssen, 92195 Meudon, France \\ 4 Observatoire de Haute-Provence, 04870 St Michel l'Observatoire, France \\ e-mail: arnold@obs-hp.fr \\ 5 Collège de France, 11 place M. Berthelot, 75321 Paris, France \\ e-mail: Labeyrie@obs-hp.fr
}

Received 23 May 2002 / Accepted 23 August 2002

\begin{abstract}
Following the idea developed in Boccaletti et al. (2000), a snapshot imaging interferometer is proposed as an alternative to the nulling interferometer for the NASA Origin project, "Terrestrial Planet Finder". This concept is based on hypertelescope, i.e. densified-pupil, imaging (Labeyrie 1996) and phase-mask coronagraphy (Rouan et al. 2000) to combine a very high angular resolution and a deep attenuation of starlight $\left(10^{-8}\right)$ as required to image extra-terrestrial planets. This article aims at presenting thorough estimations of the signal to noise ratio for different classes of stars (from F0V to M5V) and includes several sources of background noise (zodiacal and exozodiacal lights for instance). In addition, numerical simulations have been carried out and are compared to the analytical results. We find that the image of Earth-like planets can be formed with a large hypertelescope $(\sim 80 \mathrm{~m})$ in the thermal infra-red for about $73 \%$ of the stars within $25 \mathrm{pc}$.
\end{abstract}

Key words. instrumentation: interferometers - techniques: high angular resolution - methods: numerical stars: planetary systems

\section{Introduction}

Searching for Earth-like planets is the challenging goal of NASA's Terrestrial Planet Finder (TPF hereafter) space mission. The original instrument concept is based upon the idea of a nulling interferometer, according to Bracewell's option (Bracewell 1978; Bracewell \& McPhie 1979) of using a beamsplitter to produce a destructive interference in the star's wavefront received from a pair of apertures. Recent theoretical work in interferometry (Labeyrie 1996) provided us with a new concept for the instrument, the hypertelescope, for which numerical simulations and signal/noise ratio calculations have been presented in Boccaletti et al. (2000). These calculations have shown that such multi-aperture snapshot imaging interferometers outperform the former nulling interferometer concept for the search for exo-planets. Indeed, with the direct image obtained, the hypertelescope is less affected by the zodiacal and exozodiacal contamination than the nulling interferometer, in which the single-pixel detector is matched to a sub-aperture's diffraction lobe.

Send offprint requests to: P. Riaud, e-mail: pierre.riaud@obspm.fr

* The Appendix is only available in electonic form at http://www. edpsciences.org
The present article describes our recent progress in this respect. We first calculate the signal to noise ratio $(S / N$ in the following) to derive the theoretical performance of the hypertelescope with a Roddier coronagraph (Roddier \& Roddier 1997). We then show the results of more realistic numerical simulations, incorporating several sources of noise that were not accounted for in our earlier simulations (Boccaletti et al. 2000), i.e. mainly the zodiacal and exozodiacal contamination but also co-phasing errors among the sub-apertures and the thermal emission of mirrors. In addition, these simulations are performed with a new version of the phase-mask coronagraph (Rouan et al. 2000), the Four-Quadrant Phase Mask, which improves significantly the detection of faint circumstellar sources (Riaud et al. 2001) with respect to the former Roddier's phasemask (Roddier \& Roddier 1997). One of our goal was to provide a preliminary budget of errors for each source of noise to investigate the concept feasibility. Other coronagraphic masks are also applicable with densified pupil imaging like the Roddier dot plus apodized pupil first developed by Guyon \& Roddier (2000), (Guyon \& Roddier 2002). An analytical function for apodization problem can be determined rigorously with a square aperture (Aime et al. 2002) (the Prolate functions). 
The concept of the hypertelescope imaging interferometer is briefly outlined in Sect. 2 and a more practical approach of the $S / N$ is given in Sect. 3 and in the Appendix. Section 4 presents the theoretical results of exo-planet detectability and detailed numerical simulations are shown in Sect. 5. In Sect. 6, the hypertelescope concept is compared to designs using Bracewell nulling.

\section{Optical concept}

\subsection{Densified-pupil or hypertelescope imaging}

The theory and practice of densified-pupil imaging has been described in the litterature (Labeyrie 1996; Boccaletti et al. 2000; Riaud et al. 2002). An interferometer based on this concept is called a "hypertelescope". Here, we briefly review the process of image formation to illustrate the applicability and performance of hypertelescopes for stellar coronagraphy and exo-planet imaging. The general relation describing the image formation in a hypertelescope, considered as a modified Fizeau interferometer having a densified pupil where the pattern of aperture centers is preserved, is given by the following pseudoconvolution if the densification factor is large (Labeyrie 1996):

$$
I=[O \otimes I n t] * W
$$

where $I$ is the image of a point source; $O$ the object on the sky; Int is the interference function, Fourier transform of the aperture's pattern of centers; and $W(\theta)$ is the diffractive envelope or windowing function, Fourier transform of an exit sub-pupil. Unlike the infinite field extend in Fizeau interferometers (although limited by aberrations), the pupil densification shrinks the field according to $W$ while intensifying the part of the interference function appearing within the diffractive envelope. Unless a noiseless detector is used, this increases the detection sensitivity of hypertelescopes. If the sub-apertures are circular, the windowing function is an Airy pattern and the field size in the high-resolution image corresponding to the first dark ring is called the Zero Order Field $(Z O F)$. For a hexagonal periodic array, the situation considered hereafter, the $Z O F$ contains $\pi N$ resels (number of resolution elements), $N$ being the number of sub-apertures. However, the ZOF on the sky is much smaller than a sub-aperture's diffraction lobe. A point source located within this lobe but outside the ZOF still provides an interference peak inside the $Z O F$, but with a radial dispersion in polychromatic light. This happens only with periodic apertures, behaving like diffraction gratings which generate such dispersed peaks of higher order. A Higher-Order Field (HOF) larger than the $Z O F$ can thus be defined. Stellar companions located beyond the HOF can be imaged independantly with a separate densifier installed in the Fizeau image. Despite the small ZOF and HOF fields of view of hypertelescopes, terrestrial planets are potentially detectable at large angular distances as demonstrated in Sect. 4. Hypertelescopes have a substantial advantage in sensitivity of extra-solar planet detection over nulling interferometers using the beam-splitter beam combiner scheme of Bracewell.

The beam-splitter based beam combiner adopted for the original TPF concept cannot provide a direct high-resolution image. The planet's light, together with the stellar residue and the zodiacal plus exo-zodiacal emission from a sky patch matching the sub-aperture's diffraction lobe, are all received by a single detector pixel. This is less favorable for discriminating the planet than in the high-resolution image given by a hypertelescope with coronagraph since it separates spatially most of the non-planet light. The signals from zodiacal and exozodiacal light are diluted in several resels while the planet is contained in a single resel thus improving significantly the $S / N$.

\subsection{Optical scheme in the thermal IR}

The hypertelescope has several hexagonal sub-apertures located on a virtual paraboloïdal or spherical surface according to a periodic hexagonal pattern, suitable for full densification in the exit pupil. The field size being proportional to $N$, enough apertures are needed for fitting the observed planetary systems within the $Z O F$. The much wider HOF is also usable to retrieve planet images with full $S / N$ if a multi-spectral imaging camera and software are used to remove the peak dispersion. A reasonable trade-off between field size and the number of freeflyers needed involves 37 apertures. The densified pupil then resembles the aperture of a single Keck telescope, with its central obscuration removed. This optimal number $N$ is however strongly technology-dependant. If economical ways of producing and controlling small free-flyers are developed, it may become of interest to use a larger number of smaller mirrors. With a given total collecting area, the planet/zodiacal contrast in the image does not depend on $N$ or mirror sizes. For an exozodiacal cloud of size between that of the $Z O F$ and the HOF, the planet's contrast improves with $N$. This follows from the expressions of signal and noise derived below. The simulations assume 37 sub-apertures, ensuring a sufficient field of view for the $\operatorname{ZOF}$ ( $\varnothing=12 \lambda / B$ with $B$ the baseline) to image a solar system up to $25 \mathrm{pc}$ in the thermal IR. A possible optical concept is described in Labeyrie (1999).

As originally realized by Bracewell \& McPhie (1978), the emission maximum of a $300 \mathrm{~K}$ blackbody like the Earth being close to $10 \mu \mathrm{m}$, this infra-red range improves the planet/star contrast. It requires a baseline of 40 to $100 \mathrm{~m}$ to angularly separate the planet from its star. A coronagraphic device can be attached to the hypertelescope imager for attenuating the starlight and thus enhancing the visibility of the planet's image. Various novel coronagraphic schemes were proposed in the past few years for exo-planet detection (phase-mask (Roddier $\&$ Roddier 1997) or Achromatic Interfero-Coronagraph (Gay \& Rabbia 1996) for instance). In our context, we considered the Four-Quadrant Phase-Mask (FQ-PM hereafter) to be most efficient in terms of starlight rejection. The focal plane is divided into 4 equal areas with 2 quadrants providing a $\pi$ phase-shift (Rouan et al. 2000; Riaud et al. 2001) and as a result the bright star cancels out in the axial direction if its Airy disk is exactly centered on the mask. A destructive interference occurs inside the geometric pupil, where an appropriate Lyot stop can block most of the diffracted starlight. Phase-mask coronagraphy is in principle achievable with a hypertelescope since the properties of the densified-pupil image within the $Z O F$ are the same as for a monolithic telescope. 


\section{Signal to noise ratio: General relations}

The infra-red signal from an exo-planet orbiting a bright star is still very faint compared to other contributions (starlight, zodiacal and exozodiacal lights). At very close angular distances, there are two noise contributions:

the star's residual intensity and the speckle noise originating from phase aberrations in the optical train (cophasing defects or mirror roughness). In addition, extended sources (zodiacal and exo-zodiacal) spreading outside of the narrow imaging field $(Z O F)$, but within the diffraction lobe of the sub-apertures $\left(\lambda / d_{\mathrm{m}}\right.$ with $d_{\mathrm{m}}$ the diameter of the sub-aperture) contaminate the image in the narrow imaging field and therefore add photon noise.

An exo-planet peak in the densified-pupil image at $\theta$ angular distance from the star, with $J_{\mathrm{p}}$ photo-events detected per second and per resolution element (resel) is thus contaminated by:

- $J_{\mathrm{s}}(\theta)$ photon-events/s: background from the residual starlight;

- $J_{\mathrm{z}}\left(\theta, T_{\mathrm{z}}\right)$ photon-events/s: background from the zodiacal cloud with a temperature of $T_{\mathrm{z}}$;

- $J_{\mathrm{ez}}(\theta)$ photon-events/s: background from the exo-zodiacal cloud;

- $J_{\mathrm{sn}}(\theta)$ : speckle noise of the residual starlight;

- $J_{\text {id }}\left(\theta, T_{\mathrm{c}}\right)$ events/s: background from interstellar dust clouds with a temperature of $T_{\mathrm{c}}$;

- $J_{\mathrm{m}}\left(\theta, T_{\mathrm{m}}\right)$ events/s: background from the mirror optical emissivity with a temperature of $T_{\mathrm{m}}$;

- $J_{\text {conf }}$ events/s confusion noise from background stars in the $\mathrm{HOF}$;

- $N_{\text {dark }} \mathrm{e}^{-} / \mathrm{s}$ : thermal noise from the focal plane array (FPA);

$-N_{\text {ron }} \mathrm{e}^{-}$: readout noise from the FPA.

We also take into account the attenuation by the coronagraph (Fig. 1) on both the star and the planet (see Fig. 1) and also the transmission by the $Z O F$ envelope (the diffraction lobe of a sub-pupil). Each flux is calculated in a resel $\lambda / B$. In our calculation of the thermal and readout noise from the FPA we considered that each resel is sampled by four pixels (Shannon sampling). The global transmission includes a quantum efficiency of $45 \%$ for the detector (Rockwell Si:As detector), the filter transmission ( $N$ band $10.2 \pm 2 \mu \mathrm{m}$ ) and an optical transmission of $46 \%$ (based on the number of mirrors reflecting $90 \%$ ).

The expression of the $J_{\mathrm{i}}$ terms are fully detailed in the Appendix. Finally, the $S / N$ can be written as:

$S / N=\frac{J_{\mathrm{p}} \cdot t}{\sqrt{\left(\sum_{i} J_{\mathrm{i}} \cdot t+N_{\mathrm{dark}}+N_{\text {ron }}\right)}}$.

This generic relationship (Eq. (2)) is also valid for a Fizeau interferometer since it has the same planet/background contrast. In principle, the many diffraction peaks of a Fizeau interferometer can be co-added in data analysis thus providing the same $S / N$ as with a hypertelescope if a photon counting camera is used. In the general case where the detector has readout and dark current, the hypertelescope provides the same $S / N$ in a shorter time since its sensitivity is largely improved by the pupil densification which intensifies the interference peak.

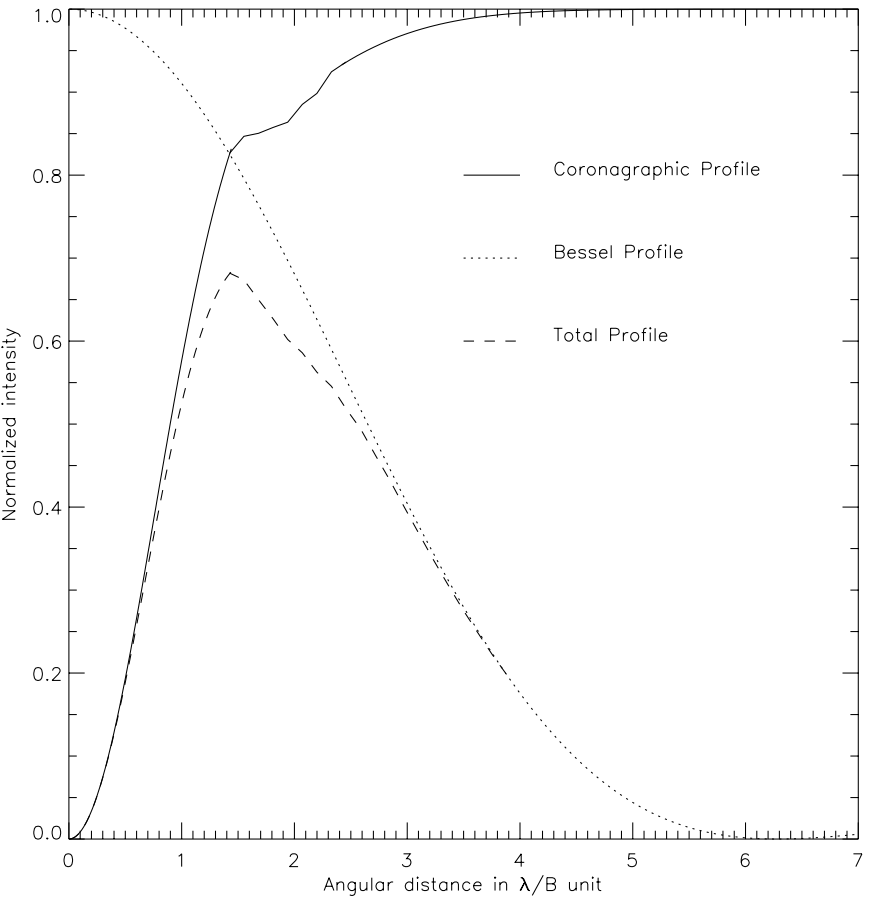

Fig. 1. Attenuation of a planet by the coronagraph (solid) and the diffractive envelope $Z O F$ (dotted) as a function of the angular separation $(\lambda / B$ unit). The maximal transmission is achieved at $1.4 \lambda / B$. However, the planet light lost from the zero-order image due to the envelope's attenuation finds its way into the first-order images appearing at known relative positions within the envelope.

\section{Signal to noise ratio: Results}

Based on Eq. (2) we investigate in this section the $S / N$ for the detection of an Earth-like planet located in the "habitable zone", i.e. with an effective temperature $T p \approx 300 \mathrm{~K}$, one of the TPF ultimate goals. As a result, the distance between the star and the planet depends on the star's temperature. Since the $Z O F$ of a hypertelescope is very small ( $\sim 12 \lambda / B$ in our concept), the spectral type must be considered to derive an accurate sample of target stars. For instance, an Earth-like planet orbiting around a G2V star located at $20 \mathrm{pc}$ lies within the $Z O F$ while the same planet around a F0V star will be twice farther away from the star, as will be the case also for a G2V star at $10 \mathrm{pc}$. As explained in Sect. 2.2, a planet lying outside the $Z O F$ still provides a small spectrum in the $Z O F$ and it is therefore important to exploit these higher-order dispersed peaks to allow the detection of terrestrial planets for any type of stars and at any distances. Table A. 2 gives the flux then remaining in the dispersed peaks when the bandwidth is reduced to accomodate the peak dispersion (in one resel) from order 0 (inside the $Z O F$ ) to order 7. As the planet moves away from the $Z O F$, the flux per resel of a higher-order peak decreases linearly owing to the spectral dispersion, while the planet's intrinsic luminosity varies as the inverse square of its distance to the observer. As a consequence, for a given spectral type, the $S / N$ of a terrestrial planet located in the HOF is larger than in the $Z O F$ (as seen in Fig. 2 for the FOV type).

Figure 2 shows the $S / N$ estimated from Eq. (2) for stars belonging to 3 spectral types (F0V, G2V and M5V) located at 


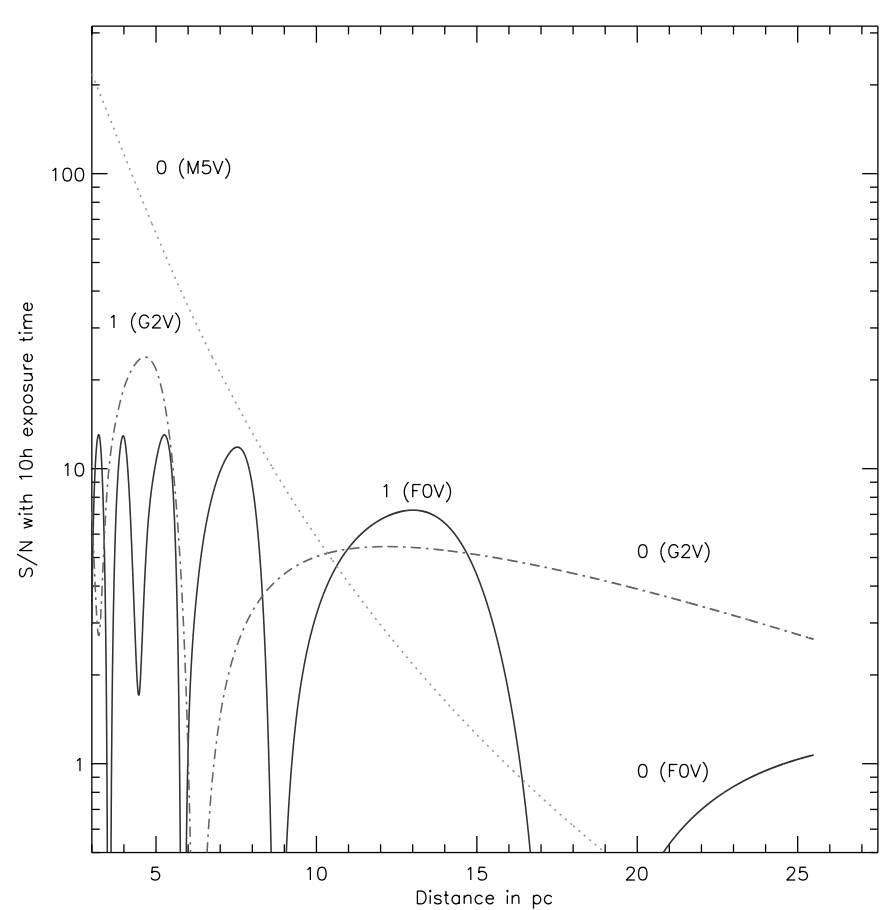

Fig. 2. Signal to noise ratio obtained in 10 hours for stars of spectral type F0V (solid), G2V (dash-dotted) and M5V (dotted) assuming a baseline of $80 \mathrm{~m}$ and versus the distance in parcsec. Earth-like planets are detectable in order 0 for M5V stars, in order 0 and 1 for G2V stars, and in orders 1 to order 5 for FOV stars.

any distance up to $25 \mathrm{pc}$. The planet peak (whatever the order of the peak) is attenuated by the windowing function towards the edge of the $Z O F$ and by the coronagraph towards the center of the field (Fig. 1), as apparent in the $S / N$ profiles presented in Fig. 2. Therefore, given the star's distance and spectral type the detection is optimal for a specific order. In the case of Fig. 2, Earth-like planets are detectable in order 0 for M5V stars, in order 0 and 1 for G2V stars, and from order 1 to order 5 for F0V stars.

We now consider a sample of 389 main-sequence stars (M5, M0, K5, K0, G5, G2, G0, F5 and F0) contained in the Hipparcos catalog (ESA, 1997) (see Fig. 3). The theoretical $S / N$ obtained at $\lambda=10 \mu \mathrm{m}$ is shown in Figs. 4 and 5 with respectively a zodiacal flux of $10.8 \mathrm{mag} /{ }^{\prime \prime 2}$ (median value) and $13.0 \mathrm{mag} /{ }^{\prime \prime 2}$ (median value at the ecliptic pole). We assume a baseline of $80 \mathrm{~m}, 10 \mathrm{~h}$ of integration time and an exo-zodiacal cloud 10 times brighter than our zodiacal cloud. As expected, the number of potentially detectable planets is considerably affected by the intensity of the zodiacal cloud. The $S / N$ can be larger than 10 for stars closer than $15 \mathrm{pc}$ and as large as $\sim 300$ for a nearby K0V star ( $\alpha$ Centauri). In the favorable case (Fig. 5), terrestrial planets could be detected around $67 \%$ of stars in our sample.

This first sample of stars is of course biased by the selection of only 9 spectral types but was useful to investigate the optimal baseline and the effect of the zodiacal light. Then to derive realistic performance, we also carried out the same calculation for any F, G, K and M main-sequence stars (667 targets) within $25 \mathrm{pc}$ with an optimal baseline of $80 \mathrm{~m}$. In Table 1, we show the full sample completude in the $N$ band (assuming each star has

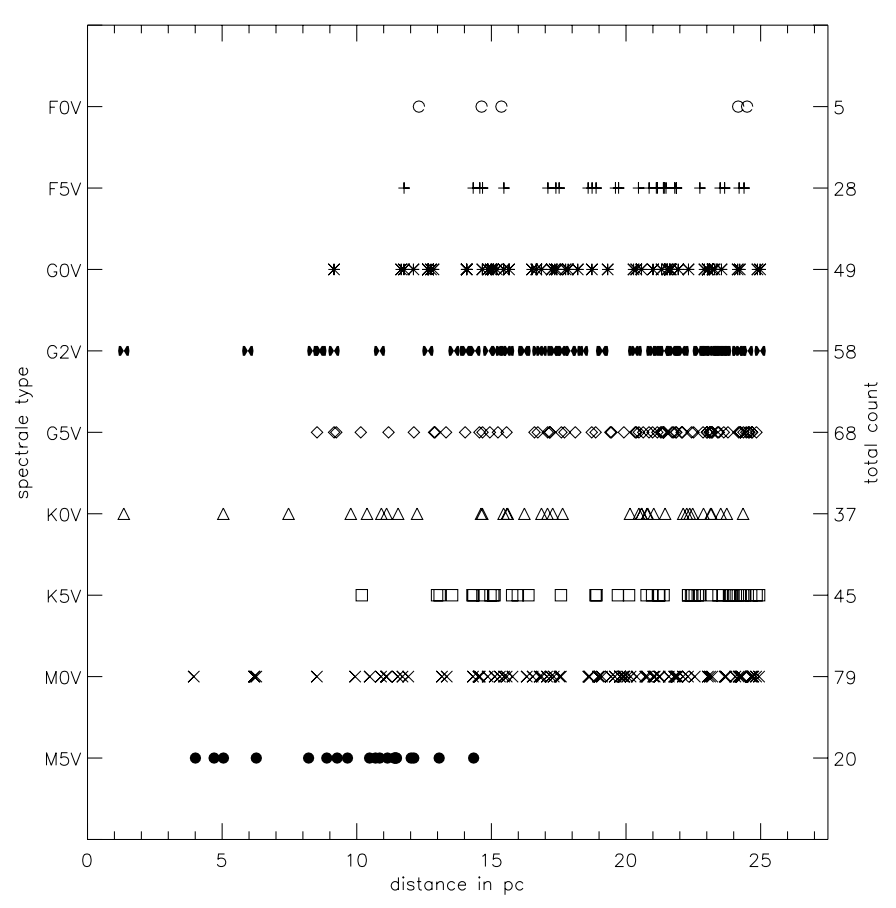

Fig. 3. Distribution of stars closer than $25 \mathrm{pc}$ for only nine spectral types (M5V to F0V). The vertical scale at left gives the spectral type and at right the total number of stars for each spectral type. For the whole sample of 389 stars, the distances and spectral types were obtained from the Hipparcos catalogue (the Hipparcos and Tycho Catalogues 1997).

Table 1. Detectivity for a full sample of stars (667) with 37 telescopes and an $80 \mathrm{~m}$ baseline. The threshold is fixed to a signal to noise ratio greater than 3 and the exposure time is 10 hours. The zodiacal flux is $13 \mathrm{mag} /{ }^{\prime 2}$ and exo-zodiacal flux is equal to 10 Zodi.

\begin{tabular}{ccccc}
\hline \hline Spectral type & F & G & K & M \\
\hline number of stars & 62 & 217 & 193 & 195 \\
detectivity & $45 \%$ & $98 \%$ & $80 \%$ & $46 \%$ \\
\hline
\end{tabular}

an Earth-like planet in orbit). Earth-like planets are potentially detectable around $73 \%$ of nearby stars in that case.

The interferometer baseline has also an important impact on the detectivity. Figure 6 shows the completude of the sample (fraction of detected planets) as a function of the baseline and a function of several intensities of the zodiacal cloud. As the flux of the zodiacal light decreases, the hypertelescope becomes obviously more sensitive to fainter stars, thus improving the sample completude. The dependance with the baseline is somewhat more complex and is correlated with the zodiacal flux. The number of positive detections reaches a maximum for an optimal baseline which depends on the zodiacal flux. For a fainter zodiacal flux $\left(L z=14.74 \mathrm{mag} /{ }^{2}\right)$, planets are becoming detectable around fainter stars, i.e. late-type stars or distant stars. However, late-type stars are most numerous and a larger baseline $(90 \mathrm{~m})$ is then required to angularly separate the terrestrial planets. The maxima of the curves is then moving towards large baselines if the zodiacal flux decreases. For instance, with $L z=13 \mathrm{mag} /{ }^{\prime \prime 2}$, the maximum of detection is achieved for 


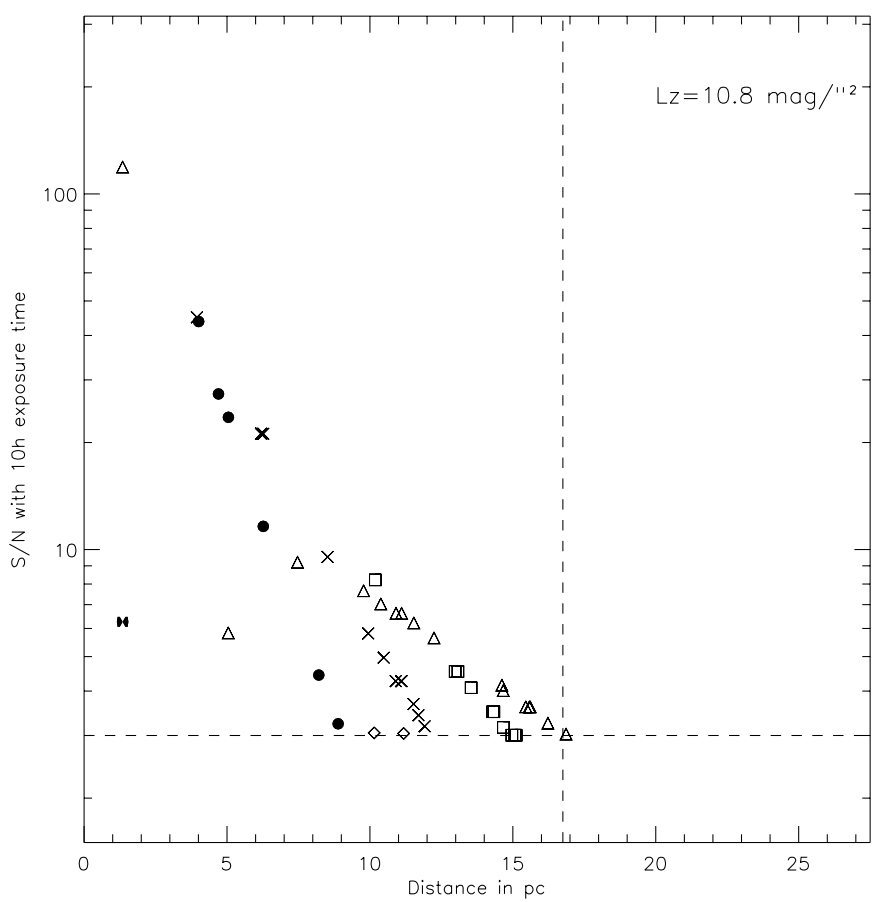

Fig. 4. Signal to noise ratio obtained for the sample of Fig. 3 with a baseline of $80 \mathrm{~m}$ and a zodiacal flux of $10.8 \mathrm{mag} /{ }^{\prime 2}$. Earth-like planets are potentially detectable around $12 \%$ of the stars ( 47 stars). The exozodiacal flux is 10 times brighter than the zodi.

a baseline of $\sim 80 \mathrm{~m}$ and for G-type and K-type stars. With a much brighter zodi $\left(L z=10.8 \mathrm{mag} /{ }^{\prime \prime 2}\right)$ the curve is almost flat because only planets around the brightest stars are detected, which means $\mathrm{F}$ and $\mathrm{G}$ stars for small baselines $(\sim 40 \mathrm{~m})$ and $\mathrm{K}$ and $\mathrm{M}$ nearby stars for large baselines $(\sim 100 \mathrm{~m})$.

Finally, we were able to estimate the most limiting source of noise in the planet's resel thanks to the calculation done in the Appendix, and we found the following noise level according to several spectral types of stars:

- Exo-Zodiacal background: $34-1450 \times J_{\mathrm{p}}$;

- Zodiacal background: $180-1080 \times J_{\mathrm{p}}$;

- Star residue: $0.7-10 \times J_{\mathrm{p}}$.

The noise level is given with respect to the star peak for a total integration of $10 \mathrm{~h}$.

\section{Numerical simulations}

Some promising numerical simulations using the principle of densified-pupil imaging have been presented in Boccaletti et al. (2000). Snapshot imaging of a solar system observed from $20 \mathrm{pc}$ was generated by combining the light from 36 small telescopes $(\varnothing=60 \mathrm{~cm}$, baseline $=100 \mathrm{~m})$ and the starlight cancellation was achieved with a phase-dot mask (Roddier \& Roddier 1997). Here we perform similar numerical simulations but using a FQ-PM to attenuate the central star. Zodiacal and exozodiacal backgrounds are included in the image together with co-phasing defects and mirror roughness. Figure 7 presents the result obtained with 37 telescopes, and $80 \mathrm{~m}$ of baseline. The phase-mask is assumed perfectly achromatic and the

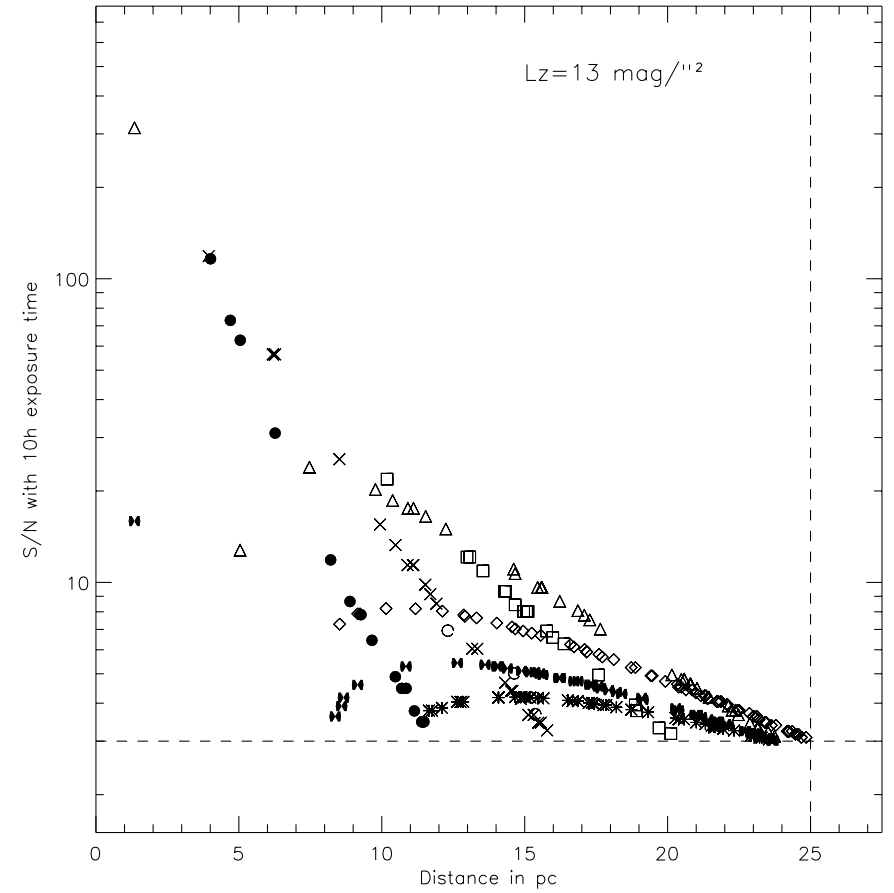

Fig. 5. Signal to noise ratio obtained for the sample of Fig. 3 with a baseline of $80 \mathrm{~m}$ and a zodiacal flux of $13.0 \mathrm{mag} /{ }^{\prime \prime 2}$. Earth-like planets are potentially detectable around $67 \%$ of the stars (262 stars). The exo-zodiacal flux is 10 times brighter than the zodi.

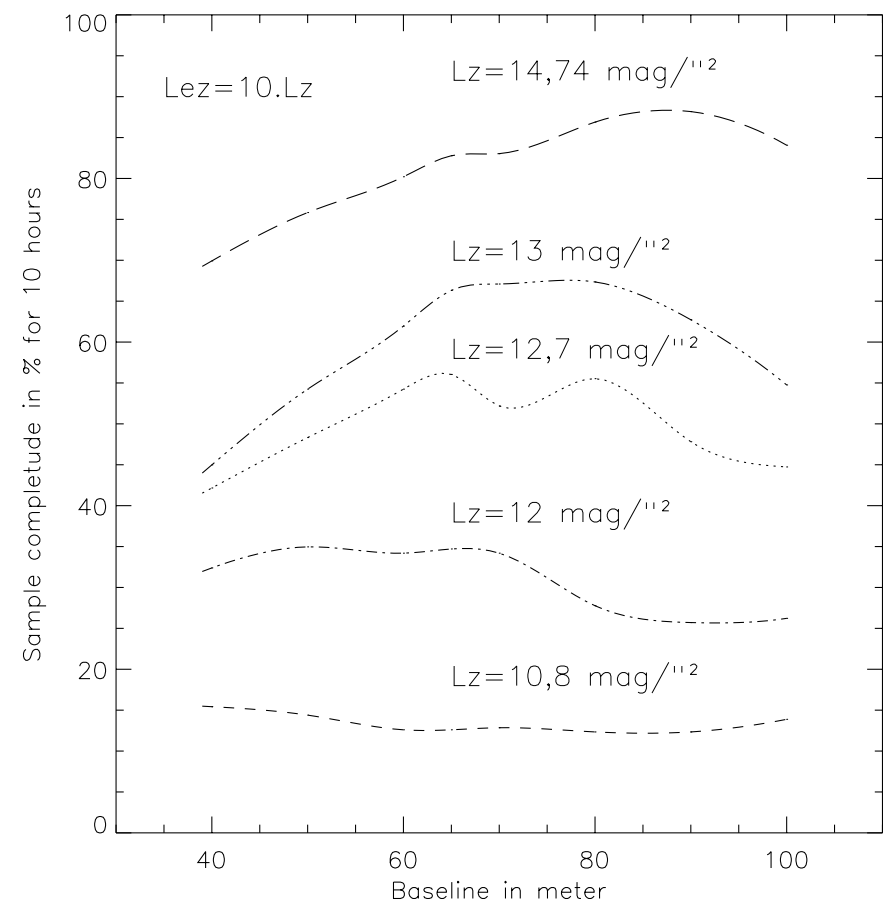

Fig. 6. Percentage of detected Earth-like planets as a function of the baseline (ranging from $40 \mathrm{~m}$ to $100 \mathrm{~m}$ ) and for a zodiacal flux of 10.8 , 12.0, 12.7, 13.0, and $14.74 \mathrm{mag} /{ }^{\prime 2}$ (Boulanger \& Pérault 1988).

interferometer has no central obscuration, which is achievable in practice by slightly off-setting the combiner optics at the focus of a large diluted mosaic mirror, whether paraboloidal or spherical. 

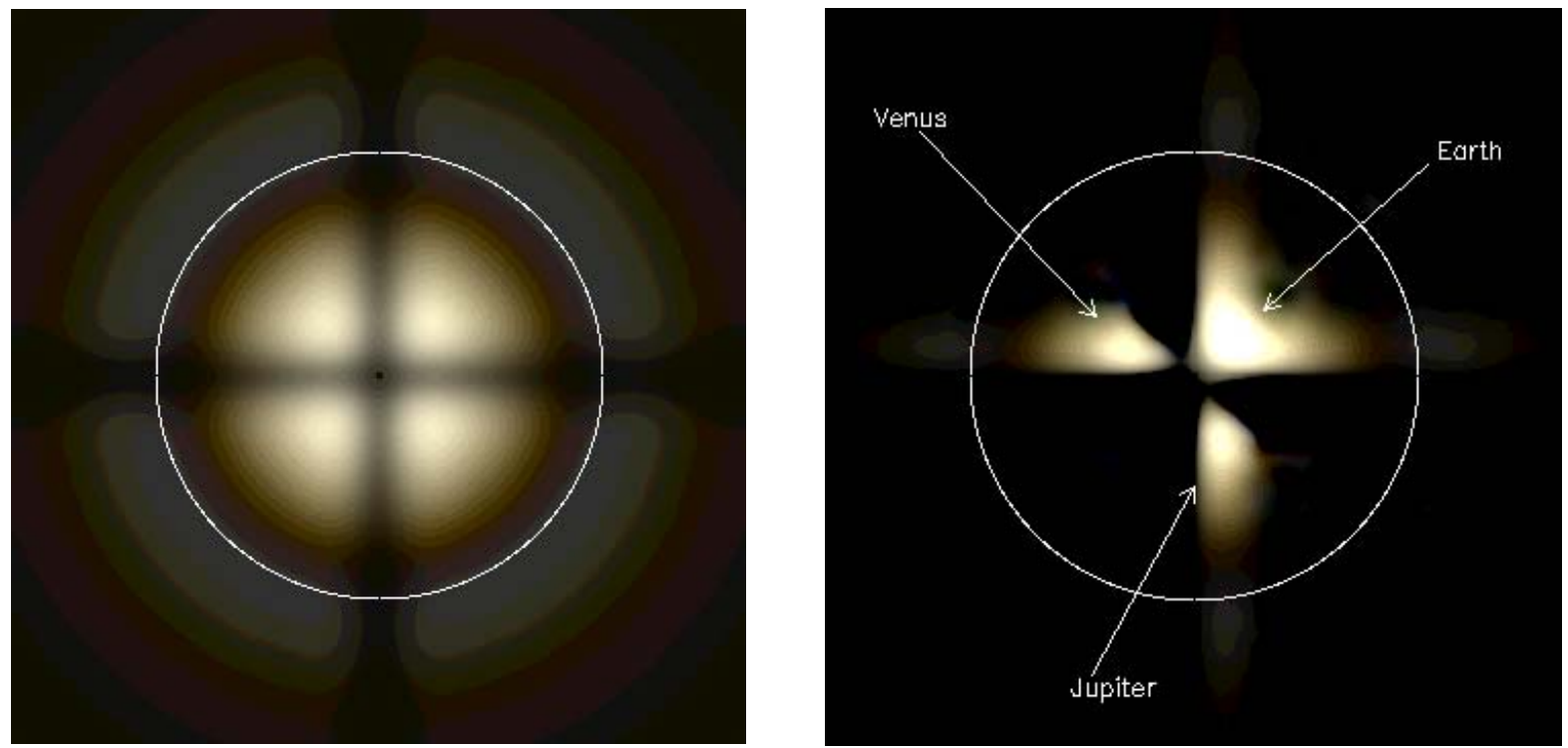

Fig. 7. Coronagraphic images obtained with a 37 aperture hypertelescope. The simulation includes photon noise $(T=10$ hours, collecting area $=10.6 \mathrm{~m}^{2}$, transmission $=46 \%$, star magnitude at $\left.10 \mu \mathrm{m}=4.7\right)$, readout noise $\left(5 \mathrm{e}^{-} / \mathrm{pixel} /\right.$ frame $)$, differential piston and tip-tilt errors between sub-apertures $(\lambda / 170 \mathrm{rms})$ and mirror roughness $(\lambda / 170 \mathrm{rms})$. The first image (left) is the coronagraphic image before subtracting the quadrants and results from a combination of 3 narrow bands $(8.4 \pm 0.75 \mu \mathrm{m}, 10.2 \pm 0.75 \mu \mathrm{m}$ and $12 \pm 0.75 \mu \mathrm{m})$. The Exo-Zodiacal light is dominant. The second image (right) shows Venus, the Earth and a secondary peak of Jupiter after subtracting opposite quadrants. The circle is the size of the Zero Order Field at $10.2 \mu \mathrm{m}$.

The central G2V star $\left(m_{v}=6.33, m_{N}=4.70\right)$ provides a photon flux of $1.64 \times 10^{6}$ events/s in the $N$ filter $(\lambda=10.2 \pm$ $2.6 \mu \mathrm{m}$ ) for a total transmission of $46 \%$. Venus, the Earth, and Mars were added in the $Z O F$, Jupiter is added in the HOF. The luminosity of the zodiacal light is $L_{\mathrm{z}}=7.7 \times 10^{-7} \mathrm{~W} /\left(\mathrm{m}^{2} \mathrm{sr} \mu \mathrm{m}\right)$ corresponding to $14.07-13-12.5 \mathrm{mag} /{ }^{\prime \prime 2}$ for respectively 8.4 $10.2-12 \mu \mathrm{m}$ (see Table A.4).

The exo-zodiacal cloud is modelled as a pair of concentric toroïdal rings with an inner diameter of $1.1 \pm 0.2 \mathrm{AU}$ and an outer diameter of $2.8 \pm 1.2 \mathrm{AU}$ (see Fig. A.2) with a total intensity of 10 times the zodiacal light. The $N$ band filter is split in three narrow-band filters $(8.4 \pm 0.75 \mu \mathrm{m}, 10.2 \pm 0.75 \mu \mathrm{m}$ and $12 \pm 0.75 \mu \mathrm{m})$. As seen in Fig. 7, the FQ-PM provides a sufficient rejection rate for the snapshot detection of Venus, the Earth and the dispersed peak of Jupiter with 37 sub-apertures in a total integration time of 10 hours. Mars would require a longer integration time (12 hours). Once compared to the simulations reported in Boccaletti et al. (2000), the FQ-PM provides a much better sensitivity than a simple phase dot for the detection of exo-planets with an hypertelescope. Moreover, the frame subtraction of a reference star is no longer required since the diffracted light from the FQ-PM is mostly centrosymmetrical. The opposite quadrants can be subtracted to remove a signicant part of symmetrical sources of noise (speckle pattern, or zodiacal and exo-zodiacal background).

\section{Comparison with the nulling interferometer}

In a multi-aperture Bracewell interferometer, the beams coming out from each sub-aperture are spatially filtered and combined with beam splitters in the pupil plane. A single detector pixel receives the combined sub-aperture lobes, containing the attenuated star and the planet. The Bracewell interferometer is adjusted to provide a central dark minimum in its angular transmission map, to attenuate the star somewhat like a coronagraph does. The nulling is obtained with appropriate phaseshifts (made achromatic if possible) among the sub-apertures. A high-resolution transmission field map can then be reconstructed by rotating the interferometer around the pointing direction. The transmission map is not uniform and we adopt an average value of $\tau \approx 0.4$ (Absil 2002). This corresponds to the highest value proposed for a Bracewell nuller. Boccaletti et al. (2000) have given analytical expressions of $S / N$ for comparing the performance of such beam-split interferometers and hypertelescopes. We now refine the comparison by using Eq. (2). In the following, the noise contributions in the Bracewell nuller (BN) are expressed as a function of the $J$ terms calculated for the hypertelescope (HT). Most of the corrective factors are geometrical. The contribution of the speckle noise $\left(J_{\mathrm{sn}}\right)$ is not considered since it is negligible.

Assuming the same collecting area for both designs, the sub-aperture radii $(r)$ are given by:

$\left(\frac{r_{\mathrm{HT}}}{r_{\mathrm{BN}}}\right)^{2}=\frac{2 \pi}{3 \sqrt{3}} \cdot\left(\frac{n_{\mathrm{BN}}}{n_{\mathrm{HT}}}\right)=\alpha \cdot\left(\frac{n_{\mathrm{BN}}}{n_{\mathrm{HT}}}\right)$

with $n_{\mathrm{BN}}$ and $n_{\mathrm{HT}}$ the number of sub-apertures for respectively the Bracewell nuller and the hypertelescope.

The planet flux now becomes:

$J_{p(\mathrm{BN})}=J_{p(\mathrm{HT})} \times \frac{\tau}{W(\theta)} \cdot \frac{A_{\mathrm{BN}}}{A_{\mathrm{HT}}} \cdot \frac{1}{E_{\text {resel }}}$

with $\tau / W(\theta)$ the ratio of planet attenuation across the field, $A_{\mathrm{BN}} / A_{\mathrm{HT}}$ the ratio of planet attenuation by the nulling system (whether a coronagraph or a nuller), and $E_{\text {resel }}$ is the fraction of the planet flux contained in one resel in the HT mode. 
The stellar level is:

$J_{\mathrm{s}(\mathrm{BN})}=J_{\mathrm{s}(\mathrm{HT})} \times \frac{\tau}{W(\theta=0)} \cdot \frac{G_{\mathrm{BN}}}{G_{\mathrm{HT}}} \cdot \frac{N_{\text {resel-HT }}}{N_{\text {resel-BN }}}$

with $G_{\mathrm{BN}} / G_{\text {coro }}$ the ratio of the star attenuation at the planet location and $N_{\text {resel }}$ indicates that the whole residual stellar flux in the field is integrated in a single pixel for the Bracewell mode. The $N_{\text {resel-HT }}$ is the number of resolution elements in the Zero Order Field of view of the HT, and $N_{\text {resel-BN }}$ is number of resolution elements for the Bracewell configuration.

To derive the contributions of extended sources in the BN mode from the HT mode, we took into account $\tau / W(\theta)$ for similar reasons as above. Also, the ratio of the sub-aperture solid angle $\left(r_{\mathrm{HT}} / r_{\mathrm{BN}}\right)^{2}$ as well as the ratio of number of resels

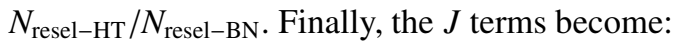

$$
\begin{aligned}
& J_{\mathrm{z}(\mathrm{BN})}=J_{\mathrm{z}(\mathrm{HT})} \times \frac{\tau}{W(\theta)} \cdot\left(\frac{r_{\mathrm{HT}}}{r_{\mathrm{BN}}}\right)^{2} \cdot \frac{\mathrm{N}_{\text {resel-HT }}}{\mathrm{N}_{\text {resel-BN }}} \\
& J_{\mathrm{ez}(\mathrm{BN})}=J_{\mathrm{ez}(\mathrm{HT})} \times \frac{\tau}{W(\theta)} \cdot\left(\frac{r_{\mathrm{HT}}}{r_{\mathrm{BN}}}\right)^{2} \cdot \frac{\mathrm{N}_{\text {resel-HT }}}{\mathrm{N}_{\text {resel-BN }}} \\
& J_{\mathrm{id}(\mathrm{BN})}=J_{\mathrm{id}(\mathrm{HT})} \times \frac{\tau}{W(\theta)} \cdot\left(\frac{r_{\mathrm{HT}}}{r_{\mathrm{BN}}}\right)^{2} \cdot \frac{\mathrm{N}_{\text {resel-HT }}}{\mathrm{N}_{\text {resel-BN }}} \\
& J_{\mathrm{m}(\mathrm{BN})}=J_{\mathrm{m}(\mathrm{HT})} \times \frac{\tau}{W(\theta)} \cdot\left(\frac{r_{\mathrm{HT}}}{r_{\mathrm{BN}}}\right)^{2} \cdot \frac{\mathrm{N}_{\text {resel-HT }}}{\mathrm{N}_{\text {resel-BN }}}
\end{aligned}
$$

while dark current and readout noise are identical:

$N_{\text {dark }(\mathrm{BN})}=N_{\text {dark }(\mathrm{HT})}$

$N_{\text {ron }(\mathrm{BN})}=N_{\text {ron }(\mathrm{HT})}$.

For the sake of simplification, let's put:

$\left(\frac{r_{\mathrm{HT}}}{r_{\mathrm{BN}}}\right)^{2} \cdot \frac{N_{\text {resel-HT }}}{N_{\text {resel-BN }}}=\alpha \approx 1.21$.

And the exposure time simply goes as the square of the ratio of $S / N$ :

$$
\begin{aligned}
& \frac{t_{\mathrm{HT}}}{t_{\mathrm{BN}}}=\left(\frac{S / N_{\mathrm{HT}}}{S / N_{\mathrm{BN}}}\right)^{2} \\
& \frac{t_{\mathrm{HT}}}{t_{\mathrm{BN}}} \approx\left(\frac{W(\theta) \cdot A_{\mathrm{HT}} \cdot E_{\mathrm{resel}}}{\tau \cdot A_{\mathrm{BN}}}\right)^{2} \\
& \times\left(\frac{J_{\mathrm{S}(\mathrm{HT})} \cdot\left(n_{\mathrm{HT}} / n_{\mathrm{BN}}\right)+\alpha \cdot(\tau / W(\theta)) \cdot \sum_{i} J_{\mathrm{i}(\mathrm{HT})}}{J_{\mathrm{s}(\mathrm{HT})}+\sum_{i} J_{\mathrm{i}(\mathrm{HT})}}\right) .
\end{aligned}
$$

Now, we derive the gain in exposure time in the $N$ band with a hypertelescope assuming the following configuration: $n_{\mathrm{HT}}=$ 37 telescopes and $n_{\mathrm{BN}}=6$ telescopes; $J_{\mathrm{z}}=13 \mathrm{mag} /{ }^{\prime \prime 2}, J_{\mathrm{ez}}=$ $10 . J_{\mathrm{z}} ; B=80 \mathrm{~m}$.

In this case the baseline of the hypertelescope is not optimized for all spectral types. However, as seen in Table 2, the gain in exposure time is significant with respect to the Bracewell nuller. The gain arises from many differents points. First, the capability of the hypertelescope to spatially separate the planet peak from the background halo owing to the angular resolution, because this interferometric configuration has a wider field of view than a Bracewell nuller. The zodiacal and exo-zodiacal light is therefore much brighter than
Table 2. Maximum gain in exposure time of the hypertelescope with respect to the Bracewell nuller for detecting a terrestrial planet, assuming $n_{\mathrm{HT}}=37, n_{\mathrm{BN}}=6, J_{\mathrm{z}}=13 \mathrm{mag} /{ }^{\prime 2}, J_{\mathrm{ez}}=10 . J_{\mathrm{z}}$ and $B=80 \mathrm{~m}$.

\begin{tabular}{ccccc}
\hline \hline Spectral type & F0V & F5V & G0V & G2V \\
\hline maximum gain & 28 & 32 & 22 & 20 \\
\hline
\end{tabular}

\begin{tabular}{cccccc}
\hline \hline Spectral type & G5V & K0V & K5V & M0V & M5V \\
\hline maximum gain & 22 & 50 & 30 & 34 & 42 \\
\hline
\end{tabular}

any exo-planets. To overcome this problem of FOV, the nuller has to be rotated to perform a synchronous detection assuming that the zodiacal and exo-zodiacal light is centrosymmetrical, unlike the planet peak. However, the observation of many disks does not confirm these possible centrosymmetrical exozodiacal clouds (see for instance the warp of $\beta$ Pic, Mouillet et al. 1997). With a synchronous detection, the Bracewell nuller does not always detect the planet contribution because the lower intensity of starlight residue in the transmission map must correspond to the planet peak position. The global efficiency with this type of detection is $1 / n_{\mathrm{BN}}$ where $n_{\mathrm{BN}}$ is the number of sub-apertures of the Bracewell interferometer. The exposure time increases with the number of apertures. However, with the hypertelescope concept we have demonstrated that behaviour is exactly the opposite. Second, the attenuation factor for the planet peak $A_{\mathrm{BN}}$ is always higher than $A_{\mathrm{HT}}$, when the nulling varies as $\theta^{4}$, however with coronagraphic device the variation is in $\theta^{2}$, where $\theta$ is the angular separation of the planet compared to the parent star.

\section{Conclusions and future work}

As already demonstrated in Boccaletti et al. (2000) the numerical simulations presented in this article confirm that a hypertelescope with coronagraph can achieve exo-planet detection in the mid-IR, and be more sensitive than interferometers using beam-splitters in their combiner. This new series of simulations bring out some additional conclusions with respect to our previous work.

First, we show that the main drawback, the field of view limitation, can be overcome if exo-planets are detected using their secondary dispersed peaks. This makes it unnecessary to vary the array size for zooming the image, as suggested before. One can define an optimal fixed baseline according to the most frequent stellar spectral types to be observed. For instance, with a baseline of $80 \mathrm{~m}$ an Earth-like planet is potentially detectable around $73 \%$ of target stars in 10 hours.

Second, the comparison between the coronagraphic hypertelescope and the Bracewell nuller has been performed in more detail than in Boccaletti et al. (2000). We similarly find that the hypertelescope can be more efficient than a Bracewell nuller by at least 1 order of magnitude, in terms of the exposure time needed to detect exo-planets (see Table 2). However, it requires more sub-apertures, the size of which is smaller if equal collecting areas are considered.

Finally, the signal to noise ratio estimation presented in the Appendix is usefull to investigate the effect of each background 
component. We find that the dominant sources of noise when searching Earth-like planets in the mid-IR are the exo-zodiacal and zodiacal clouds. The stellar residuals, with the speckles generated by phase errors are fainter if the starlight rejection is larger than $10^{5}$.

Phase-mask coronagraphy is well suited to hypertelescopes. Some ongoing experiment are addressing the technology readiness of these devices. Miniature versions of these have been tested at Observatoire de Haute-Provence, both in the laboratory and on the sky. They use diffractive Pedretti et al. (2000) or geometric-optics Gillet et al. (2002, submitted) methods of pupil densification. A four-quadrant phase mask coronagraph is also being tested. Some of us are also involved in the NGST European consortium for the study of a phase-mask coronagraph to be implemented in the imager of MIRI (mid-IR instrument) (Dubreuil et al. 2002), and obviously the issues for NGST and TPF are very similar. A first prototype was manufactured for visible wavelengths and succesfully tested on an optical bench allowing starlight rejection $\approx 45000$ in the central resel (Riaud et al. to be published) and a contrast as large as $10^{6}$ at $3 \lambda / d$. A simple phase mask, having a chromatic response, is used but achromatized versions were also recently proposed (Riaud et al. 2001 for instance) and are being studied in our group.

A subsequent step which we are preparing is the testing of a hypertelescope with a phase-mask coronagraph. Given the sensitivity gain with respect to previous forms of nulling, a hypertelescope version of the TPF should now be considered, and more detailed comparisons can be made. The direct imaging capability is also of obvious interest for a broad range of science, including the deep-field imaging of remote galaxies. Because deployable structures will be difficult to use for arrays larger than 50 or $100 \mathrm{~m}$, we further explore the use of free-flyer elements driven by solar sails. Solar sails are attractive in terms of accuracy but require low masses (of less than $25 \mathrm{~kg})$. The hypertelescope concept with its small mirror elements $(60-70 \mathrm{~cm})$ seems to be adapted to this technology. Sails also serving as multi-layer sunshields can keep the mirror elements below 40-60 K. For cost reasons, one can start with a modest hypertelescope having 7 mirror elements and then later add sub-apertures to increase the field of view and the full sample completude.
Acknowledgements. We wish to thank the DARWIN team: Alain Léger principal investigator, Alain Labèque chief enginer, Marc Ollivier and Pedrag Sékulic for helpful discussions. And also we wish to thank the referee Olivier Guyon for useful comments and corrections.

\section{References}

Absil, O. 2001 Proc. From Optical to Millimetric Interferometry, Liège Int. Astroph. Coll., 79

Aime, C., Soummer, R., \& Ferrari, A. 2002, A\&A, 389

Beichman, C., \& Velusamy, T. 1997, BAAS, 29, 1310

Beichman, C., Lindensmith, C., \& Woolf, N. J. 1999, The Terrestrial Planet Finder, JPL Publication, 99-3

Boccaletti, A., Riaud, P., Moutou, C., \& Labeyrie, A., 2000, Icarus, $145,2,628$

Boulanger, F., \& Pérault, M. 1988, ApJ, 330, 964

Bracewell, R. N. 1978, Nature, 274, 780

Bracewell, R. N., \& McPhie, R. H. 1979, Icarus, 38, 136

Dubreuil, D., et al. 2002, Proc. of SPIE 2002, submitted

Gay, J., \& Rabbia, Y. 1996 , CR. Acad. Sci. Paris, 332, Serie II b, 265

Gillet, S., Riaud, P., Dejonghe, J., Lardière, O., et al. 2002, A\&A, to appears

Guyon, O., \& Roddier, F. 2000, Proc. SPIE, 4006

Guyon, O., \& Roddier, F. 2002, A\&A, 391, 379

The Hipparcos and Tycho Catalogues, 1997, ESA SP-1200

Labeyrie, A. 1996, A\&AS, 118, 517

Labeyrie, A. 1999, in Cargèse summer school Planets Outside the Solar System: Theory and Observations, ed. J. M. Mariotti, \& D. Alloin, NATO ASI, 532, 261

Léger, A., Mariotti, J. M., Mennesson, B., et al. 1996, Icarus, 123, 249

Leinert, Ch., Bowyer, S., Haikala, L. K., Hanner, M. S., et al. 1997, A\&AS, 127, 1

Mouillet, D., Larwood, J. D., Papaloizou, J. C. B., \& Lagrange, A. M. 1997, MNRAS, 292, 896

Pedretti, E., Labeyrie, A., Arnold, L., et al. 2000, A\&AS, 147, 285

Roddier, F., \& Roddier, C. 1997, PASP, 109, 815

Rouan, D., Riaud, P., Boccaletti, A., Clénet, Y., \& Labeyrie, A. 2000, PASP, 112, 1479

Riaud, P., Boccaletti, A., Rouan, D., Lemarquis, F., \& Labeyrie, A. 2001, PASP, 113, 1145

Riaud, P., Gillet, S., Labeyrie, A., Boccaletti, A., et al. 2001 Proc. From Optical to Millimetric Interferometry, Liège Int. Astroph. Coll., 85 